\title{
Genomic divergence between nine- and three-spined sticklebacks
}

\author{
Baocheng Guo ${ }^{1 *}$, Frédéric JJ Chain², Erich Bornberg-Bauer ${ }^{3}$, Erica H Leder $^{4}$ and Juha Merilä
}

\begin{abstract}
Background: Comparative genomics approaches help to shed light on evolutionary processes that shape differentiation between lineages. The nine-spined stickleback (Pungitius pungitius) is a closely related species of the ecological 'supermodel' three-spined stickleback (Gasterosteus aculeatus). It is an emerging model system for evolutionary biology research but has garnered less attention and lacks extensive genomic resources. To expand on these resources and aid the study of sticklebacks in a phylogenetic framework, we characterized nine-spined stickleback transcriptomes from brain and liver using deep sequencing.

Results: We obtained nearly eight thousand assembled transcripts, of which 3,091 were assigned as putative oneto-one orthologs to genes found in the three-spined stickleback. These sequences were used for evaluating overall differentiation and substitution rates between nine- and three-spined sticklebacks, and to identify genes that are putatively evolving under positive selection. The synonymous substitution rate was estimated to be $7.1 \times 10^{-9}$ per site per year between the two species, and a total of 165 genes showed patterns of adaptive evolution in one or both species. A few nine-spined stickleback contigs lacked an obvious ortholog in three-spined sticklebacks but were found to match genes in other fish species, suggesting several gene losses within 13 million years since the divergence of the two stickleback species. We identified 47 SNPs in 25 different genes that differentiate pond and marine ecotypes. We also identified 468 microsatellites that could be further developed as genetic markers in nine-spined sticklebacks.

Conclusion: With deep sequencing of nine-spined stickleback cDNA libraries, our study provides a significant increase in the number of gene sequences and microsatellite markers for this species, and identifies a number of genes showing patterns of adaptive evolution between nine- and three-spined sticklebacks. We also report several candidate genes that might be involved in differential adaptation between marine and freshwater nine-spined sticklebacks. This study provides a valuable resource for future studies aiming to identify candidate genes underlying ecological adaptation in this and other stickleback species.
\end{abstract}

Keywords: Pungitus pungitius, Gasterosteus aculeatus, Comparative genomics, Transcriptome, Substitution rate, Adaptive evolution

\section{Background}

The rapid advances in sequencing technologies have facilitated the development of comparative genomics an important approach in contemporary evolutionary biology research [1,2]. The stickleback fishes (Gasterosteidae) provide an excellent model system for such comparative studies. The three-spined stickleback (Gasterosteus aculeatus) has become a vertebrate

\footnotetext{
* Correspondence: baochengguo@gmail.com

${ }^{1}$ Ecological Genetics Research Unit, Department of Biosciences, University of Helsinki, Helsinki, Finland

Full list of author information is available at the end of the article
}

'supermodel' allowing a combination of studies at molecular, developmental, phenotypic, and population genetic levels to explore factors and processes relevant for adaptive evolution in ecologically relevant contexts $[3,4]$. The three-spined stickleback is a small teleost populating diverse ecosystems across a wide geographic distribution in the northern hemisphere and occurs in marine, brackish, and freshwater habitats. Populations that have colonized freshwater habitats after the retreat of Pleistocene ice sheets have evolved remarkable morphological and behavioral diversity as compared to marine populations $[5,6]$. For example, they have repeatedly

\section{Biomed Central}


evolved changes in body shape, skeletal armor, trophic apparati, pigmentation, osmoregulatory functions, life history, and behavior [5]. The genetic architecture for several of these phenotypic adaptations has been - or is being - deciphered [7-15]. Interestingly, the parallel evolution (similar phenotypes evolving independently in different populations derived from a common ancestor) of armor loss, pelvic reduction, and pigmentation has been found to result from parallel genetic changes in similar genes $[8,9,11,14]$. However, relatively little is known about the genetics of these or other traits in other stickleback species (but see: [16-20]).

The nine-spined stickleback (Pungitius pungitius) is an emerging model for evolutionary biology research [21] and has diverged from the three-spined stickleback around 13 million years ago (Mya) [22], but the two species are ecologically - and to some degree also phenotypically - very similar [23]. Phylogeographic and population genetic analyses of the nine-spined stickleback demonstrate that their colonization and adaptation to freshwater habitats from marine environments has occurred independently multiple times [24-26]. Meanwhile, freshwater nine-spined sticklebacks have also evolved - repeatedly and independently - similar morphological [26-28], behavioral [29,30], neurological [31-36], and physiological [37,38] phenotypes in different localities. Notably, similar adaptive traits also have been evolved in parallel between nine- and three-spined sticklebacks [6]. For example, both marine nine- and threespined sticklebacks have a complete pelvis, but several different freshwater populations in both species have undergone a genetically based reduction - or even total loss - of the pelvic girdle and associated spines [16-18]. However, it is still uncertain whether or not the genetic underpinnings of the pelvic reduction in nine- and three-spined sticklebacks are the same. For instance, Shapiro et al. [16] first suggested that changes of Pitx1 expression might contribute to pelvic reduction in both species, but later discovered that the major loci controlling for pelvic development were completely different between the two species. This suggests that the pelvic reduction in these species is an example of genetic convergence [17] (but see [18]). Hence, nine- and threespined sticklebacks offer a powerful opportunity to study whether or not similar phenotypic changes across species are associated with the same genes or genetic mechanisms.

A genome-wide comparative study can help us to better understand how selection has shaped divergence and illuminate the genetic basis of parallel evolution in nine- and three-spined sticklebacks. It can also reveal the extent of genome-wide differentiation across protein-coding and non-coding regions and the prevalence of species-specific genes that may influence the evolu- tionary trajectory of divergent species. However, compared to the three-spined stickleback with abundant genomic resources $[3,39,40]$, genomic resources for the nine-spined stickleback are still largely lacking (but see: $[17,41,42])$. For example, development of microsatellite markers for study of nine-spined stickleback currently is based on the three-spined genome, but cross-species utility of microsatellite primers is limited due to low amplification success [43]. Fortunately, the recent explosion of affordable Next Generation Sequencing (NGS) technology provides evolutionary and ecological researchers a great opportunity to conduct genome-wide studies of non-model organisms with limited genetic and genomic resources [44-46]. For instance, transcriptome, a collection of expressed sequences, represents a sample of the spatiotemporally expressed genome that can be used for comparative genomic studies at an interspecific level, as well as genetic diversity analyses at an intraspecific level. Here, we used deep sequencing (454 GS FLX) to characterize partial brain and liver transcriptomic libraries of nine-spined sticklebacks from marine and freshwater populations exhibiting a high degree of morphological and genetic divergence [26-38,41]. With the resulting transcripts, we (1) characterized the sequence divergence between the two closely related stickleback species, (2) investigated rates of molecular evolution for patterns consistent with positive selection, and (3) evaluated sequence differentiation between marine and freshwater nine-spined sticklebacks.

\section{Results}

\section{Sequencing and assembly}

We obtained a total 337,630 high quality reads with mean length of 250 bp from 454 sequencing of four cDNA libraries from nine-spined sticklebacks (Additional file 1: Figure S1). Contig assembly of the reads were combined from the four cDNA libraries into one "nine-spined stickleback transcriptome" containing 7,932 contigs $\geq 100$ bp (median $=403$ bp, Additional file 1: Figure S2) with an average coverage depth of 38 reads (Additional file 2: Table $\mathrm{S} 1$ ).

\section{Functional annotation}

A BLASTX search returned 3,347 (42.2\%) nine-spined stickleback contigs with significant hits to three-spined stickleback genes. This proportion of contigs with BLAST hits is similar to previous transcriptome studies [47-49], in which contigs without significant hits may consist of untranslated transcripts, chimeras or assembly artifacts. Blast2Go with the Gene Ontology (GO) annotations database was used for further annotation and 2,071 contigs have one or more GO terms (Additional file 1: Figure S3). We additionally found that 104 contigs had no significant BLASTX hit with protein sequences 
from the three-spined stickleback but had significant hits with protein sequences in at least one of the other seven fish genomes available from Ensembl. By using BLASTN and BLAT searches, we confirmed that 15 of the 104 contigs had no hits in the current three-spined stickleback genome (Additional file 2: Table S2). Because these contigs correspond to genes in other teleost genomes, this suggests that the orthologous sequences of these contigs have probably been lost in the three-spined stickleback rather than gained in nine-spined sticklebacks.

\section{Sequence comparison between nine- and three-spined sticklebacks}

We found that 3,091 out of the 3,347 nine-spined stickleback contigs $(92.4 \%)$ had a pairwise $K_{s} \leq 0.5$ compared to their three-spined stickleback orthologs (Figure 1), and these had an average length of $690 \mathrm{bp}$ (Additional file 1: Figure S4). We restricted all further analyses to these 3,091 contigs, or "unigenes", in an attempt to curtail the effects of erroneously called orthologs with large $K_{s}$ values. The corresponding genes are more or less evenly distributed across the three-spined stickleback genome with $2.3 \%$ to $7.1 \%$ of genes on each chromosome, and the gene number per chromosome is positively correlated with chromosome size $\left(r_{s}=0.84\right.$, $P<1.2 \times 10^{-6}$, in Additional file 1: Figure S5). Given the conserved genomic synteny between the two species [35], these observations suggest that the unigenes are a relatively unbiased sample of nine-spined stickleback genes in terms of genomic distribution.

We used three methods to detect positive selection on genes in sticklebacks. We first calculated the pairwise

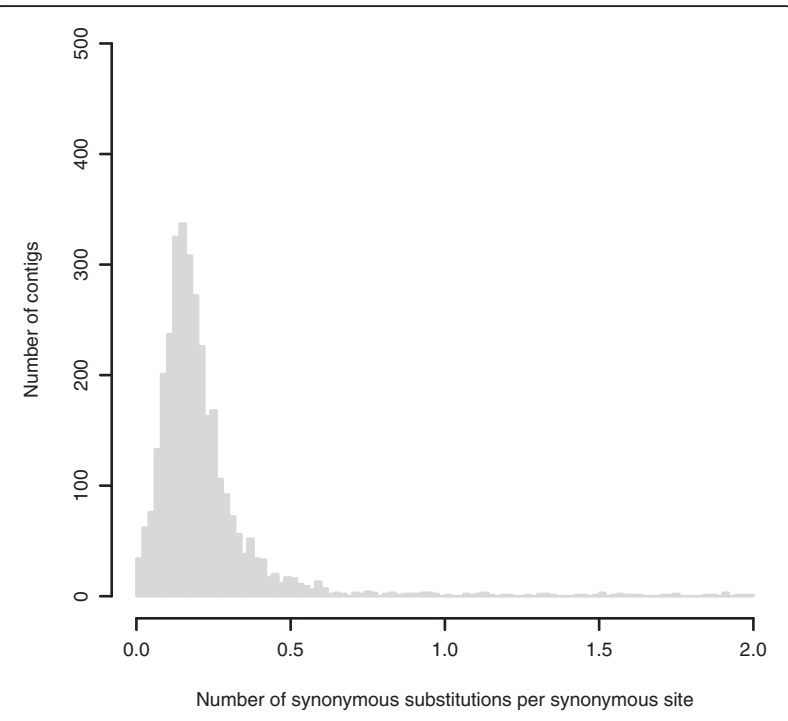

Figure 1 Distribution of $K_{s}$ distances between nine-spined stickleback contigs and their three-spined stickleback orthologs. substitution rates $K_{s}, K_{a}$, and $K_{a} / K_{s}$ between the ninespined stickleback unigenes and their putative orthologs in the three-spined stickleback (Figure 2). Genes are generally under strong purifying selection (low $K_{a} / K_{s}$ values), with a mean pairwise $K_{s}$ value was $0.1841 \pm$ 0.0017 (mean \pm SD). A total of 194 (6.3\%) orthologous pairs had a $K_{a} / K_{s}$ ratio between 0.5 and 1 (points above the grey line in Figure 2), and $74(2.4 \%)$ had a $K_{a} / K_{s}$ ratio $>1$ (points above the black line in Figure 2). The latter 74 unigenes are distributed across 16 chromosomes (Additional file 3: Table S3).

We also performed the branch-site test with medaka as an outgroup to detect positive selection operating on sites along each stickleback lineage. The branch-site test revealed a total of 33 unigenes $(p<0.05$, eight after multiple test correction with q-value $<0.05)$ that are putatively evolving under positive selection in the ninespined stickleback lineage and 39 unigenes (seven after multiple test correction) in the three-spined stickleback lineage (Additional file 3: Table S4). We also found 82 unigenes (37 after multiple test correction) with sites evolving under positive selection in the ancestral lineage before the split between nine- and three-spined sticklebacks (Additional file 3: Table S4).

A third method was used for inferring positive selection by utilizing nine-spined stickleback SNPs. We analyzed the patterns of selection among genes with the MK test and the direction of selection (DoS). We found 48 unigenes that departed from neutrality (Chi-square test with Yates correction, $\mathrm{df}=1, p<0.05), 18$ of which show a signature of positive selection (Additional file 3 :

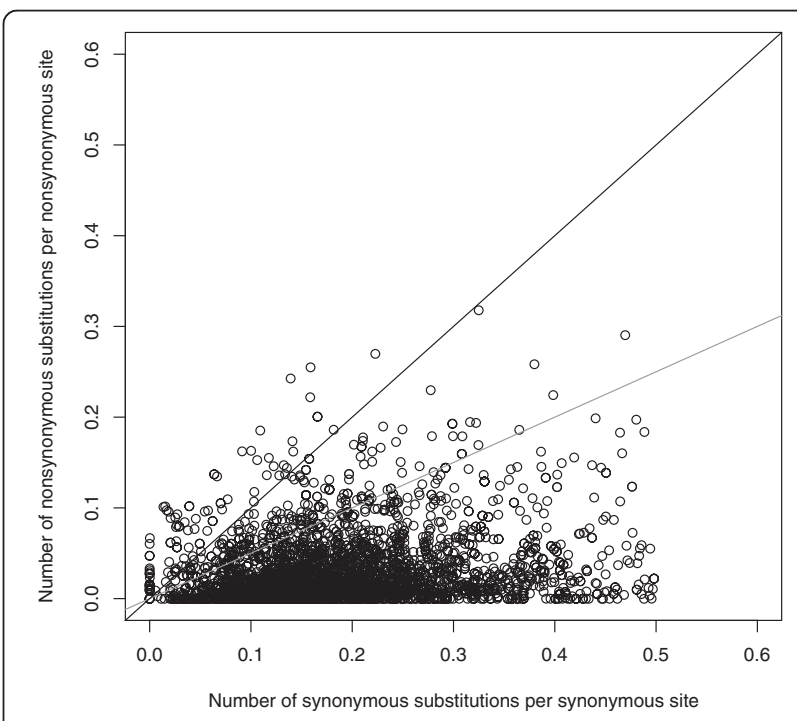

Figure 2 Distribution of pairwise $K_{a}$ and $K_{s}$ distances between nine-spined stickleback unigenes and their three-spined stickleback orthologs. Genes with $K_{a} / K_{s}$ ratio $>1$ fall above the black line while those with $K_{a} / K_{s}$ ratio between 0.5 and 1 fall between the gray and black lines. 
Table S5). However, none of these signatures remained statistically significant after correction for multiple tests.

It is noteworthy that positive selection on seven genes was detected by at least two of the three methods mentioned above. For example, two genes with a pairwise $K_{a} / K_{s}$ ratio $\geq 1$ that are involved in lipid transport are also detected using the branch-site test, of which one gene (apolipoprotein B - ENSGACG00000009637) is consistent with positive selection in the nine-spined stickleback lineage and the other gene (a vitellogenin gene - ENSGACG00000009711) is consistent with positive selection in the three-spined stickleback lineage. Other overlaps from methods of detecting positive selection include a gene (adenylate cyclase 6 - ENSGACG 00000008575) detected by the MK test and the branchsite test in the nine-spined stickleback lineage, and four genes (complement factor H-related 3 - ENSGACG000 00001733, fetuin B - ENSGACG00000005690, HECT domain containing E3 ubiquitin protein ligase 1 - ENSG ACG00000012853 and an uncharacterized gene - ENS GACG00000007507) detected by both pairwise $K_{a} / K_{s}$ and the MK test. Combining all three tests, we found a total of 165 genes with patterns of adaptive evolution in either the nine- or three-spined stickleback, or both. These genes are distributed rather evenly across all of the three-spined stickleback chromosomes except XIV (Additional file 1: Figure S5). We found 126 of these 176 genes with associated GO annotations spanning a broad range of functions (Figure 3). We found that nine GO terms (viz. translational elongation, macromolecule biosynthesis, protein biosynthesis, translation, cellular biosynthesis, physiological process, macromolecule metabolism, biosynthesis, and energy derivation by oxidation of organic compounds) were significantly overrepresented among these 165 genes by comparing to all three-spined stickleback genes, which suggested that these 165 genes have been subject to adaptive evolution (family-wise error rate, $P<0.05$; Additional file 3: Table 56 ).

A total of 368 nine-spined stickleback unigenes contained partially sequenced UTRs $\geq 50 \mathrm{bp}$. The average K2P distance of these UTRs and their three-spined stickleback orthologous sequence was $0.0709 \pm 0.0020$ (median $=0.0688$ ), whereas the average $\mathrm{K} 2 \mathrm{P}$ distance of the coding regions for these same genes was $0.0513 \pm$ 0.0017 (median $=0.0436$ ). The average pairwise $K_{s}$ for the 368 unigenes was $0.1746 \pm 0.0047$ (median $=0.1637$ ) and is close to that of the all 3,091 unigenes (mean = $0.1841 \pm 0.0017$; median $=0.1687$ ), which suggests no bias of the 368 unigenes with UTR information, at least with respect to $K_{s}$. The divergence of UTRs was significantly higher as compared to the divergence in corresponding coding regions (Wilcoxon signed rank test, $P<2.2 \times 10^{-16}$ ) but significantly lower than that of $K_{s}$ (Wilcoxon signed rank test, $P<2.2 \times 10^{-16}$ ), which suggests that UTRs have evolved under lower selective pressures than coding regions, albeit not neutrally (assuming that synonymous sites evolve close to neutrally). Based on the divergence estimates above and the species divergence time of 13 Mya [22], we calculated the substitution rate as $2.0 \times 10^{-9}$ per site per year in coding regions

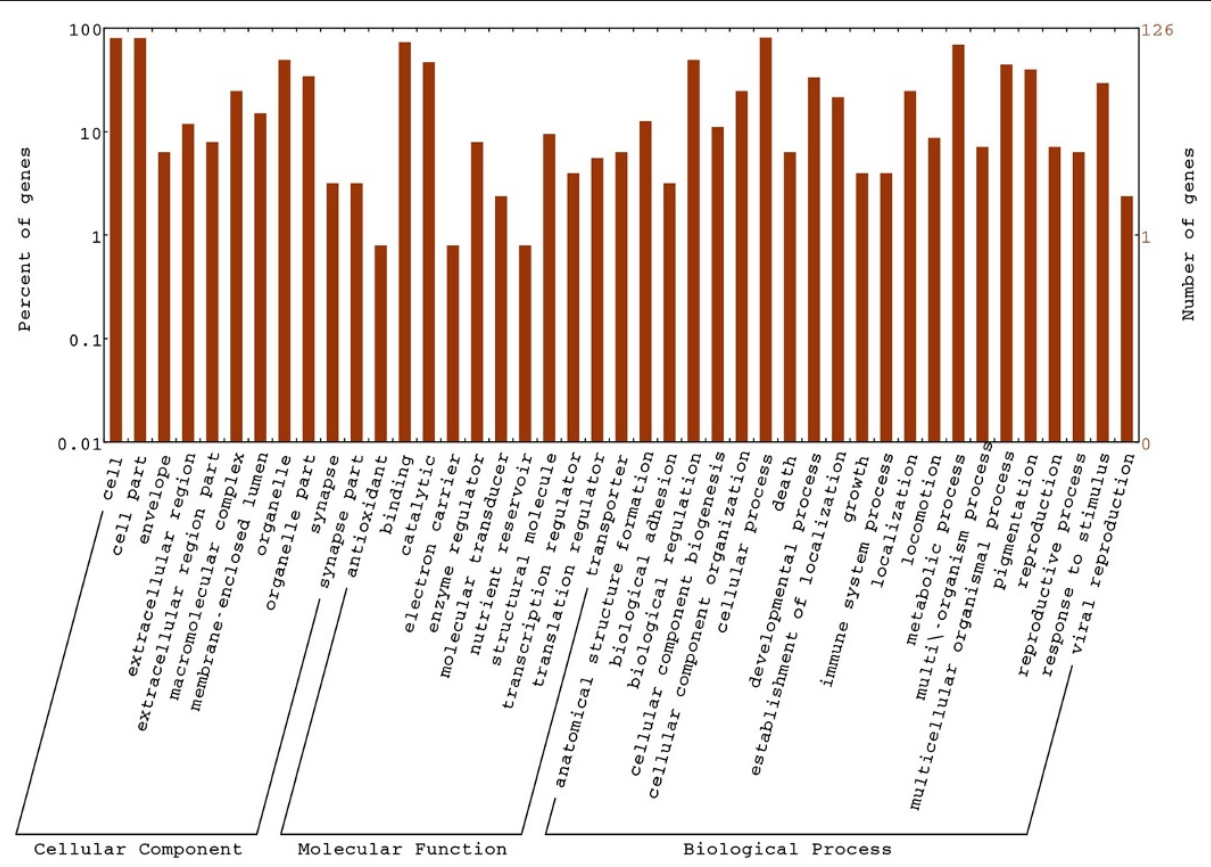

Figure $3 \mathrm{GO}$ assignment for genes showing adaptive evolution between nine- and three-spined sticklebacks. GO annotation were retrieved using Blast2GO followed by classification and plotting with WEGO [93]. 
(including both synonymous and nonsynonymous sites) and $2.7 \times 10^{-9}$ per site per year in UTRs between nineand three-spined sticklebacks.

\section{Divergence between marine and freshwater nine-spined sticklebacks}

We found 1,814 SNPs (0.044\% of evaluated genic sites) among 718 unigenes in the sampled nine-spined sticklebacks (934 unique SNPs in the marine sample, 642 unique SNPs in the pond sample, and 238 SNPs shared in common). Many of the SNPs (567) are predicted to be nonsynonymous changes, while the remaining are either synonymous (665) or in UTRs (582) (Additional file 3: Table S7). We found 47 SNPs in 28 unigenes (spanning 25 different genes) that lead to 'fixed' genotypes between the two ecotypes, including 17 homozygous differences. These divergent SNPs occur in both tissue types and as such are not tissue-specific differences but most probably reflect general genetic differences between the ecotypes (at least from the sampled individuals). Of the fixed homozygous differences, five are nonsynonymous SNPs, ten are synonymous SNPs and two are SNPs found in UTRs (Additional file 3: Table S8).

\section{Discovery of microsatellite markers}

Microsatellites are important genetic markers for nonmodel organisms and have been widely used for studies of nine-spined sticklebacks $[18,19,24,41,43]$. We analyzed the nine-spined stickleback unigenes to identify microsatellite markers. We obtained 468 SSRs in 358 unigenes (Additional file 3: Table S9). In terms of abundance, dinucleotide repeats were most abundant (178, $38.0 \%)$ followed by trinucleotide repeats (148, 31.6\%), mononucleotide repeats $(139,29.7 \%), 1$ tetranucleotide repeat, and 2 hexanucleotide repeats. Of the 468 SSRs, 428 are perfect and 40 are compound. AC/GT (124 out of $178,69.7 \%$ ) was the most abundant dinucleotide repeat motif and AGG/CTT (58 out of 148, 39.2) was the most abundant trinucleotide repeat motif.

\section{Discussion}

\section{The nine-spined stickleback transcriptome}

In recent years, the use of comparative genomic approaches in a phylogenetic framework has shed much light on a variety of fundamental evolutionary questions, such as adaptive evolution [3,50,51], genetic variation [52-55], and speciation [56-58]. Development of genomic resources is the first step towards such biological questions. Using 454 pyrosequencing, we have contributed to the improvement of genomic resources for nine-spined sticklebacks. We provide over three thousand transcript sequences that correspond to an orthologous gene in the three-spined stickleback, and report hundreds of genic microsatellites that can be used as markers in future experiments with nine-spined sticklebacks. The data provided here significantly increase the number of available gene sequences for nine-spined sticklebacks since there are currently fewer than 1,000 sequence entries in the National Center for Biotechnology Information. Given its status as an emerging model for evolutionary biology research [21], this transcriptomic data will be of interest to researchers investigating the evolution of nine-spined sticklebacks, for example by using the identified SNPs or microsatellite markers for population genetics studies. It also allows for more refined inferences concerning stickleback and teleost evolution in a phylogenetic framework by providing orthologs of closely related fish species. Thus, apart from contributing a large number of new gene sequences to the research domain, the results of this study represent the first reported nine-spined stickleback transcriptomic resource, and as such, provide a starting point for intraand inter-specific genomic comparisons in sticklebacks.

\section{Sequence divergence between nine- and three-spined sticklebacks}

The nine-spined stickleback transcriptome characterized in this study allowed us to survey sequence divergence between two closely related species - nine- and threespined sticklebacks. Because the two species diverged 13 Mya [22], we anticipated that the genetic differences would be considerable despite the highly ecological, phenotypic, and genetic similarities between the species $[23,43]$. The rate of sequence substitution is of central importance to understand mechanisms underlying molecular evolution. Rates of nonsynonymous and synonymous substitutions are good indicators of selective pressures at the sequence level of protein-coding genes $[59,60]$. Synonymous sites usually evolve neutrally and can provide insights on the background rate of sequence evolution $[59,60]$, thus we used the $K_{s}$ values of proteincoding genes to estimate neutral substitution rates in sticklebacks. The average substitution rate was estimated to be $7.1 \times 10^{-9}$ per synonymous site per year between nine- and three-spined sticklebacks (Additional file 1: Figure S6) when calibrated to the divergence time of 13 Mya. This rate is faster than previously published genome-wide substitution rate estimates available across mammals $\left(2.2 \times 10^{-9}\right.$ per synonymous site per year; [61]), but is nearer the substitution rate of teleosts $\left(1.25 \times 10^{-6}\right.$ in cichlids; [46]) as the rates of molecular evolution in fish are known to be fast compared to other vertebrates [62]. Additionally, the unigenes we identified may be enriched with highly expressed genes that are more easily detected in transcriptomic sequencing, and thus the estimated substitution rate might be an underestimation because highly expressed protein coding genes usually 
evolve slowly [63]. Nevertheless, this estimated substitution rate should be a useful yardstick for research in teleost molecular evolution in general, and particularly for those studies on stickleback phylogeny and molecular clock dating.

Identifying genes that show evidence of positive selection can help us in understanding whether closely related species occupying similar ecological niches share genetic attributes involved in adaptation. The $K_{a} / K_{s}$ ratio (= 1: neutral evolution; $>1$ : positive selection; $<1$ : purifying selection) is often used for diagnosing the extent and direction of selection on sequence evolution $[59,60]$. Using three analyses based on nonsynonymous and synonymous substitutions, a total of 165 genes show indications of positive selection in one or both species of sticklebacks. These 165 genes have significantly smaller pairwise $K_{s}$ (Wilcoxon-Mann-Whitney test, $P=1.4 \times$ $10^{-7}$ ) but significantly larger pairwise $K_{a}$ (WilcoxonMann-Whitney test, $P=5.7 \times 10^{-4}$ ) compared to the other analyzed genes (Additional file 1: Figure S7). Despite a broad range of $\mathrm{GO}$ annotations that these genes are involved with, we found that they showed enrichment in several functional categories. Such genes may be of particular interest for further studies aiming to investigate their detailed functions, as well as possible associations with ecological differences between stickleback species.

In addition to coding sequence changes, regulatory sequence changes may play an important role in repeated adaptive evolution of freshwater three-spined sticklebacks [3]. In general, UTRs, especially 3'-UTRs, are found to evolve neutrally among very closely related taxa [46]. However, we found that UTRs between nine- and three-spined sticklebacks are under stronger purifying selection as compared to synonymous sites, but under more relaxed selection as compared to coding regions (both synonymous and nonsynonymous sites). These findings suggest that some UTRs may be important in shaping stickleback evolution [3].

Gene gains and losses are important processes contributing to evolutionary innovation and differentiation $[64,65]$, perhaps especially so in teleosts because of the teleost-specific whole genome duplication event [66]. The comparison between stickleback orthologs revealed that some genes are likely to have been lost in the threespined stickleback, as they exist both in nine-spined sticklebacks and other model fish genomes. It is also possible that these genes are missing from the current three-spined stickleback genome assembly, or that the genes have evolved so rapidly that they no longer resemble the same gene in other fishes. Of the genes that might have been lost in three-spined sticklebacks, nine have associated GO terms related to binding (protein and iron), cell migration, and membrane component.
However, a more complete grasp of the number of genes differentially lost and retained between nine- and threespined sticklebacks can only be answered with a complete nine-spined stickleback genome. Nevertheless, our results suggest that as in the case of other vertebrates [65,67], stickleback divergence is also accompanied with gene losses.

However, we are aware that our results largely depend on the initial dataset for which we can make comparisons between genes. Because we used a subset of all genes in the genome, we cannot capture the entire list of variation and genes that are evolving under positive selection. In fact, our dataset may further be biased towards slowly evolving genes under stronger purifying selection if we are capturing mainly highly expressed genes, and those with low $K_{s}$ values. Nevertheless, our results should provide a useful first step towards unraveling the genetics underlying divergence between nine- and three-spined sticklebacks. Taken together, our analyses of substitution rates, positive selection and gene loss suggest that there are considerable genetic differences between these two ecologically and phenotypically similar species.

\section{Genetic divergence between marine and freshwater nine-spined sticklebacks}

Much research has been directed towards investigating genome-wide divergence between marine and freshwater three-spined sticklebacks and many genes associated with their divergence have been identified $[3,68]$. Genetic differentiation between marine and freshwater ninespined sticklebacks also has been described in studies utilizing microsatellites [41] and restriction-site-associated DNA sequencing [42]. For example, Shikano et al. [41] found several functionally- and physiologicallyimportant genes that had experienced divergent selection between different habitats, and Bruneaux et al. [42] showed that genomic regions enriched for genes having functions related to immunity, chemical stimulus response, lipid metabolism, and signaling pathways had experienced positive selection. However, in-depth genome-wide studies of genetic differentiation between marine and freshwater nine-spined sticklebacks have been lacking. Here, we probed the genome-wide genetic differentiation between marine and freshwater ninespined sticklebacks to understand whether similar or different genetic changes underlying divergence between freshwater and marine populations exist in the two stickleback species. We found 25 genes with 'fixed' genotypes between marine and freshwater nine-spined sticklebacks (Additional file 3: Table S8), and these represent candidates for ecotypic differentiation in nine-spined sticklebacks. Interestingly, one of these genes, the enolase 1a (ENSGACG00000007396) gene has also been 
found to be associated with the divergence of marine and freshwater three-spined sticklebacks [3]. ATPases are another group of interesting genes that have been associated with the marine and freshwater divergence in sticklebacks. We found that the ATP5B and ATP6v1ba genes have SNPs differentiating marine and freshwater nine-spined sticklebacks, and similar evidence is available from ATP6V1Aa [41] in nine-spined and ATP6VOA1 and ATP6VOE1 in three-spined sticklebacks [3]. Furthermore, a transferrin gene (ENSGACG0000 0013533) with a putative function in iron ion transport may be of particular interest for understanding adaptive population divergence of marine and freshwater ninespined sticklebacks, since ion concentration is one of the notable environmental differences demarcating marine and freshwater habitats. Hence, enolase 1a, ATP5B, $A T P 6 v 1 b a$ and transferrin provide promising candidates for further investigations focused on understanding the molecular mechanisms of differentiation and adaptation between marine and freshwater stickleback populations. Further studies screening more populations and individuals are needed to evaluate the robustness of these results, as well as to understand how often adaptive divergence between marine and freshwater populations of different stickleback taxa is occurring through evolution in the same or in different genes or genetic elements.

\section{Conclusions}

With the massively parallel pryrosequencing of ninespined stickleback cDNA libraries, we identified over three thousand unique gene transcripts and hundreds of genic microsatellites. Using these transcripts, we calculated sequence substitution rates in coding regions, in UTRs, and across synonymous sites between nine- and three-spined sticklebacks. We identified over a hundred genes with molecular patterns of positive selection in one or both stickleback lineages and found several candidate genes that might be involved in differential adaptation between marine and freshwater nine-spined sticklebacks. Both the same and different genes were found to associate with marine and freshwater divergence across stickleback taxa. Apart from these specific findings, the study brings about significant amount of new resources (viz. gene sequences, microsatellites, and SNPs) to the reach of the research community interested in fish and stickleback genomics in particular.

\section{Methods}

This study did not involve human subjects, and our experimental protocol was approved by the ethics committee of National Animal Experiment Board, Finland (permission numbers: ESLH-STSTH223A and STH037A).

\section{Fish sampling, RNA isolation, and cDNA library construction}

We sampled two male and two female nine-spined sticklebacks from the Baltic Sea (Helsinki, Finland; $60^{\circ} 12^{\prime} \mathrm{N}$, $25^{\circ} 11^{\prime} \mathrm{E}$ ), and one male and one female from an isolated freshwater pond (Rytilampi, Finland; $66^{\circ} 23^{\prime} \mathrm{N}, 19^{\circ} 19^{\prime} \mathrm{E}$ ). We chose to sequence the brain and liver transcriptomes to access a large number of diverse transcripts, as these are highly complex organs with complex transcriptomes. Total RNA was extracted from brain and liver tissues using TRIzol reagent (Invitrogen, Carlsbad, CA, USA) according to the manufacturer's protocol. We constructed four cDNA libraries (marine brain, marine liver, freshwater brain, and freshwater liver) with the SuperScript $^{\oplus}$ Double-Stranded cDNA Synthesis Kit (Invitrogen, cat. no. 11917-010), according to the manufacturer's protocol. Equimolar amounts of the total RNA from each of the two males and two females from marine population were pooled for construction of the marine brain library, but only one male and one female were used for the marine liver library. Likewise, RNA from one male and one female were used for the freshwater brain and liver libraries.

\section{Transcriptome sequencing and assembly}

We barcoded the four cDNA libraries and sequenced them in a half plate of GS FLX (Roche 454) Standard Chemistry run by DNA Sequencing and Genomics Laboratory, Institute of Biotechnology, University of Helsinki at Helsinki, Finland. Sequences have been deposited in the NCBI Short Read Archive (Accession no. SRR846896, SRR846899, SRR846900, and SRR846901). We trimmed adaptors and low quality reads using custom Perl scripts. We then assembled the cleaned reads using v2.5.3 of the GS De Novo Assembler [69] into contigs representing four transcriptomic libraries, one brain and one liver library from each population. We obtained three additional transcriptomic libraries by pooling the contigs from the four cDNA libraries into a marine transcriptome, a freshwater transcriptome and the allencompassing 'nine-spined stickleback transcriptome'. These transcriptomic libraries were assembled from reads using a minimum overlap length of $40 \mathrm{bp}$ and a minimum overlap identity of $98 \%$. Detailed information of the transcriptome assemblies are listed in Additional file 2: Table S1.

\section{Gene annotation}

Our annotations focused on the 'nine-spined stickleback transcriptome' that was assembled with all reads combined from the four cDNA libraries. We only included assembly contigs with a minimum length of $100 \mathrm{bp}$ for further analyses and used two comprehensive methods to annotate the remaining contigs. We first assigned 
their putative identities using BLASTX [70] searches against protein datasets of the three-spined stickleback reference (a freshwater individual [3]) from the Ensembl database [71] (release-68) with an E-value cutoff of $1 \times 10^{-10}$, and paired the contigs with their top BLAST hit. The resulting gene pairs are herein referred to as orthologs. Importantly, because of varying transcript lengths and alternative transcription, different ninespined stickleback contigs can map to different regions or to alternative transcripts of the same three-spined stickleback gene. To identify genes that are possibly lost (or missing) from the three-spined stickleback genome, we used contigs without hits against three-spined stickleback proteins as queries in BLASTX searches against protein datasets of the other model fishes Danio rerio, Gadus morhua, Oreochromis niloticus, Oryzias latipes, Takifugu rubripes, and Tetraodon nigroviridis from the Ensembl database release-68 and Xiphophorus maculatus from the Ensembl database release-70. We then used those contigs with hits in other model fish as queries in BLASTN and BLAT [72] searches against the three-spined stickleback genome to validate that these putative genes are lost (or missing) from the threespined stickleback genome.

We assigned putative functions for each selected ninespined stickleback contig using version 2.5.0 of Blast2GO [73], which performs a BLASTX search against the non-redundant database from NCBI with default parameters. We obtained annotated accession numbers and Gene Ontology (GO) [74] numbers from NCBI QBLAST [70] based on an E-value of $1 \times 10^{-10}$ and a high-scoring segment pair cut-off greater than 33 . We conducted the annotation procedure with the following parameters: a pre-E-value-Hit-Filter of $10^{-6}$, a proSimilarity-Hit-Filter of 15 , an annotation cut-off of 55 , and a GO weight of 5 . GO term enrichment test was conducted using GOSSIP [75].

To obtain putative protein-coding and amino acid sequences, we employed GeneWise2 [76] to deduce the open reading frame for each contig sequence using its corresponding best-match protein in the three-spined stickleback as a guide. The putative untranslated region (UTR) of each contig was obtained based on the results of the ORF prediction and further assessed by alignment with UTRs of their corresponding putative orthologs using MUSCLE [77] with default settings to avoid including assembly artifacts.

\section{Substitution rate estimation}

We aligned the amino acid sequences of each pair of orthologs from nine- and three-spined sticklebacks using MUSCLE [77] with default settings and manually inspected for possible alignment artifacts. We performed DNA sequence alignments from the resulting protein alignments using a custom Perl script. The number of nonsynonymous substitutions per nonsynonymous site $\left(K_{a}\right)$ and synonymous substitutions per synonymous site $\left(K_{s}\right)$ between each orthologous pair was computed using a maximum-likelihood method [78] with the YN00 program implemented in PAML version 4.4 [79]. Only ninespined stickleback contigs with $K_{s} \leq 0.5$ compared to their three-spined stickleback orthologs were selected for further analyses (e.g., GO annotation and SNP calling) and are referred to as unigenes. In addition, if different nine-spined stickleback contigs aligned to the same three-spined gene, nine-spined stickleback contig with smallest $K_{s}$ to the three-spined gene was kept; in case two contigs aligned to the same three-spined gene with equal $K_{s}$ values, we randomly kept one of them for further analysis. As mentioned previously, several ninespined stickleback contigs or unigenes can correspond to different regions or transcripts of the same threespined stickleback gene.

We estimated the overall substitution rate between the nine- and three-spined stickleback genomes based on the divergence between unigenes and their orthologs (coding region and UTRs at least $50 \mathrm{bp}$ long) while considering a divergence time around 13 Mya [22]. Distances of coding regions and UTRs were calculated separately using Kimura's two parameter (K2P) model [80] in EMBOSS [81].

We performed the branch-site test $[82,83]$ with the codeml program in PAML [79] to detect positive selection operating on sites in the nine- and three-spined stickleback lineages. For this test, we used the corresponding 1-to-1 ortholog in O. latipes (determined from Ensembl) as an outgroup. We were able to perform this test for 2,458 unigenes. We calculated the $P$ values based on the Chi-square critical values of 3.84 (5\%) as recommended in PAML [79]. Multiple test correction was performed using the qvalue package in $\mathrm{R}$ [84] with default settings to correct for the false discovery rate (FDR).

\section{SNP calling}

To determine single nucleotide polymorphisms (SNPs) among sampled nine-spined stickleback individuals, we mapped all of the cleaned reads from each of the four cDNA libraries separately to the nine-spined stickleback unigenes using BWA-SW in BWA 0.6.1 [85] with default settings. SNPs from each of the four mappings were called using samtools 0.1.18 [86] with mpileup -I to disable indel calling as insufficient flushing during 454 sequencing usually leads to indel events around homopolymers [87]. Only bases with a Phred quality score of at least 20 were considered for the SNP calling. Combined with the three-spined stickleback ortholog, SNPs were used for performing the McDonald-Kreitman (MK) test of neutral evolution [88] using libsequence [89]. The 
MK test is used for evaluating the ratio of polymorphism (intraspecies differences) and divergence (interspecies differences) at nonsynonymous and synonymous sites. Under neutrality, the ratio of polymorphism and divergence at these site classes is equal. We calculated an unbiased estimator of the direction of selection (DoS) developed by Stoletzki \& Eyre-Walker [90], which is a modification of the neutrality index (NI) [91] by calculating the difference between the proportion of divergent and polymorphic nonsynonymous substitutions. Whereas DoS is zero under neutrality, positive selection driving an excess of nonsynonymous divergence between species would render DoS positive, and purifying selection reflected by an excess of nonsynonymous polymorphisms within species would decrease DoS below zero. Statistical significance in the departure from neutrality for each gene was determined by the Chi-square test with Yates correction as implemented in libsequence [89].

\section{Microsatellite identification}

We used a microsatellite identification program - MISA [92] to identify microsatellite motifs in our nine-spined unigenes. We searched for all types of Simple Sequence Repeats (SSRs) from mononucleotide to hexanucleotides using the following parameters: at least 10 repeats for mono-, 6 repeats for di- and 5 repeats for tri-, tetra-, penta- and hexanucleotide for simple repeats. We identified both perfect (SSRs containing a single repeat motif) and compound (SSRs composed of two or more motifs separated by $<100$ bp) SSRs.

\section{Availability of supporting data}

The data sets supporting the results of this article are available in the in the National Center for Biotechnology Information Short Read Archive repository (Accession no. SRR846896, SRR846899, SRR846900, and SRR846901), and included within the article and its additional files.

\section{Additional files}

Additional file 1: Figure S1. Read length distribution; Figure S2. Contig length distribution; Figure S3. Unigene GO annotation plotting with WEGO; Figure S4. Unigene length distribution; Figure S5.

Chromosomal distribution of unigenes along the three-spined stickleback genome (grey) and genes with molecular patterns of adaptive evolution between nine- and three-spined sticklebacks (black); Figure S6. Gene-specific substitution rates per synonymous site per year between nine- and three-spined sticklebacks; Figure S7. Boxplot of pairwise $K_{a}$ and $K_{s}$ between nine- and three-spined sticklebacks of the 165 genes showing evidence for adaptive evolution and other genes.

Additional file 2: Table S1. Assembly summary of nine-spined stickleback transcriptomic libraries; Table S2. Genes putatively lost in threespined sticklebacks.

Additional file 3: Table S3. Results from pairwise $\mathrm{Ka} / \mathrm{Ks}$ calculation for detection of positive selection between nine- (9SS) and three-spined
(3SS) sticklebacks; Table S4. Results from a branch-site test for positive selection; Table S5. Direction of Selection (DoS) from the MK test; Table S6. GO term enrichment test of the genes with adaptive evolution between nine- and three-spined sticklebacks using GOSSIP; Table S7. Genes with SNPs between ecotypes; Table S8. Fixed genotype differences between ecotypes; Table S9. SSRs in unigenes of nine-spined stickleback.

\section{Competing interests}

The authors declare that they have no competing interests.

\section{Authors' contributions}

BG and JM conceived the study. EL performed the experiments. BG and FC analyzed the data. BG, FC, EB, and JM wrote the paper. All authors have read and approved the final manuscript.

\section{Acknowledgements}

We thank Gabor Herczeg and Abigel Gonda for help in obtaining the samples and Marika Karjalainen for help with the lab work. We thank CSC - the Finnish IT Center for Science for computing support. We also thank three anonymous reviewers for their constructive comments. Our research was supported by Academy of Finland (grant numbers 250435 and 265211 to J.M.).

\section{Author details}

${ }^{1}$ Ecological Genetics Research Unit, Department of Biosciences, University of Helsinki, Helsinki, Finland. ${ }^{2}$ Max Planck Institute for Evolutionary Biology, Department of Evolutionary Ecology, Plön, Germany. ${ }^{3}$ Institute for Evolution and Biodiversity, Evolutionary Bioinformatics, Westfalian Wilhelms University of Münster, Münster, Germany. ${ }^{4}$ Division of Genetics and Physiology,

Department of Biology, University of Turku, Turku, Finland.

Received: 3 May 2013 Accepted: 31 October 2013

Published: 5 November 2013

\section{References}

1. Hardison RC: Comparative genomics. PloS Biol 2003, 1:e58.

2. Stapley J, Reger J, Feulner PG, Smadja C, Galindo J, Ekblom R, Bennison C, Ball AD, Beckerman AP, Slate J: Adaptation genomics: the next generation. Trends Ecol Evol 2010, 25:705-712.

3. Jones FC, Grabherr MG, Chan YF, Russell P, Mauceli E, Johnson J, Swofford R Pirun M, Zody MC, White $S$, et al: The genomic basis of adaptive evolution in threespine sticklebacks. Nature 2012, 484:55-61.

4. Gibson G: The synthesis and evolution of a supermodel. Science 2005, 307:1890-1891.

5. McKinnon JS, Rundle HD: Speciation in nature: the threespine stickleback model systems. Trends Ecol Evol 2002, 17:480-488.

6. Bell MA, Foster SA: The evolutionary biology of the threespine stickleback. London: Oxford University Press; 1995.

7. Chan YF, Marks ME, Jones FC, Villarreal G, Shapiro MD, Brady SD, Southwick AM, Absher DM, Grimwood J, Schmutz J, et al: Adaptive Evolution of pelvic reduction in sticklebacks by recurrent deletion of a Pitx1 Enhancer. Science 2010, 327:302-305.

8. Miller CT, Beleza S, Pollen AA, Schluter D, Kittles RA, Shriver MD, Kingsley DM: cis-regulatory changes in kit ligand expression and parallel evolution of pigmentation in sticklebacks and humans. Cell 2007, 131:1179-1189.

9. Colosimo PF, Hosemann KE, Balabhadra S, Villarreal G, Dickson M, Grimwood J, Schmutz J, Myers RM, Schluter D, Kingsley DM: Widespread parallel evolution in sticklebacks by repeated fixation of ectodysplasin alleles. Science 2005, 307:1928-1933.

10. Peichel CL, Ross JA, Matson CK, Dickson M, Grimwood J, Schmutz J, Myers RM, Mori S, Schluter D, Kingsley DM: The master sex-determination locus in threespine sticklebacks is on a nascent Y chromosome. Curr Biol 2004 14:1416-1424.

11. Colosimo PF, Peichel CL, Nereng K, Blackman BK, Shapiro MD, Schluter D, Kingsley DM: The genetic architecture of parallel armor plate reduction in threespine sticklebacks. PloS Biol 2004, 2:635-641.

12. Shapiro MD, Marks ME, Peichel CL, Blackman BK, Nereng KS, Jonsson B, Schluter D, Kingsley DM: Genetic and developmental basis of evolutionary pelvic reduction in threespine sticklebacks. Nature 2004, 428:717-723. 
13. Peichel CL, Nereng KS, Ohgi KA, Cole BLE, Colosimo PF, Buerkle CA, Schluter $D$, Kingsley DM: The genetic architecture of divergence between threespine stickleback species. Nature 2001, 414:901-905.

14. Cresko WA, Amores A, Wilson C, Murphy J, Currey M, Phillips P, Bell MA, Kimmel CB, Postlethwait JH: Parallel genetic basis for repeated evolution of armor loss in Alaskan threespine stickleback populations. Proc Natl Acad Sci USA 2004, 101:6050-6055.

15. Kimmel CB, Ullmann B, Walker C, Wilson C, Currey M, Phillips PC, Bell MA, Postlethwait JH, Cresko WA: Evolution and development of facial bone morphology in threespine sticklebacks. Proc Natl Acad Sci USA 2005, 102:5791-5796.

16. Shapiro MD, Bell MA, Kingsley DM: Parallel genetic origins of pelvic reduction in vertebrates. Proc Natl Acad Sci USA 2006, 103:13753-13758.

17. Shapiro MD, Summers BR, Balabhadra S, Aldenhoven JT, Miller AL, Cunningham CB, Bell MA, Kingsley DM: The genetic architecture of skeletal convergence and sex determination in ninespine sticklebacks. Curr Biol 2009, 19:1156-1156.

18. Shikano T, Laine VN, Herczeg G, Vilkki J, Merilä J: Genetic architecture of parallel pelvic reduction in ninespine sticklebacks. G3- Genes Genom Genet 2013, 3:1833-1842.

19. Laine VN, Shikano T, Herczeg G, Vilkki J, Merilä J: Quantitative trait loci for growth and body size in the nine-spined stickleback Pungitius pungitius L. Mol Ecol. in press.

20. Laine VN, Shikano T, Herczeg TG JV, Merilä J: QTL analysis of behavioral traits in nine-spined sticklebacks (Pungitius pungitius). Behav Genet. in press.

21. Merilä J: Nine-spined stickleback (Pungitius pungitius): an emerging model for evolutionary biology research. Ann N Y Acad Sci 2013 1289:18-35.

22. Bell MA, Stewart JD, Park PJ: The world's oldest fossil threespine stickleback fish. Copeia 2009, 2009:256-265.

23. Wootton RJ: The biology of the sticklebacks. New York: Academic; 1976

24. Shikano T, Shimada Y, Herczeg G, Merilä J: History vs. habitat type: explaining the genetic structure of European nine-spined stickleback (Pungitius pungitius) populations. Mol Ecol 2010, 19:1147-1161.

25. Teacher AGF, Shikano T, Karjalainen ME, Merilä J: Phylogeography and genetic structuring of European nine-spined sticklebacks (Pungitius pungitius)-mitochondrial DNA evidence. PLoS One 2011, 6:e19476.

26. Aldenhoven JT, Miller MA, Corneli PS, Shapiro MD: Phylogeography of ninespine sticklebacks (Pungitius pungitius) in North America: glacial refugia and the origins of adaptive traits. Mol Ecol 2010, 19:4061-4076.

27. Herczeg G, Gonda A, Merilä J: Evolution of gigantism in nine-spined sticklebacks. Evolution 2009, 63:3190-3200.

28. Herczeg G, Gonda A, Merilä J: Morphological divergence of NorthEuropean nine-spined sticklebacks (Pungitius pungitius): signatures of parallel evolution. Biol J Linnean Soc 2010, 101:403-416.

29. Herczeg G, Gonda A, Merilä J: Predation mediated population divergence in complex behaviour of nine-spined stickleback (Pungitius pungitius). J Evol Biol 2009, 22:544-552.

30. Herczeg G, Gonda A, Merilä J: The social cost of shoaling covaries with predation risk in nine-spined stickleback, Pungitius pungitius, populations. Anim Behav 2009, 77:575-580.

31. Gonda A, Herczeg G, Merilä J: Habitat-dependent and-independent plastic responses to social environment in the nine-spined stickleback (Pungitius pungitius) brain. Proc R Soc B-Bio/ Sci 2009, 276:2085-2092

32. Gonda A, Herczeg G, Merilä J: Adaptive brain size divergence in ninespined sticklebacks (Pungitius pungitius)? J Evol Biol 2009, 22:1721-1726.

33. Gonda A, Välimäki K, Herczeg G, Merilä J: Brain development and predation: plastic responses depend on evolutionary history. Biol Lett 2012, 8:249-252.

34. Trokovic N, Herczeg G, Ab Ghani NI, Shikano T, Merilä J: High levels of fluctuating asymmetry in isolated stickleback populations. BMC Evol Biol 2012, 12:115.

35. Trokovic N, Herczeg G, Scott McCairns R, Izza Ab Ghani N, Merilä J: Intraspecific divergence in the lateral line system in the nine-spined stickleback (Pungitius pungitius). J Evol Biol 2011, 24:1546-1558.

36. Gonda A, Herczeg G, Merilä J: Population variation in brain size of nine-spined sticklebacks (Pungitius pungitius)-local adaptation or environmentally induced variation? BMC Evol Biol 2011, 11:75.

37. Shimada Y, Shikano T, Kuparinen A, Gonda A, Leinonen T, Merilä J: Quantitative genetics of body size and timing of maturation in two nine-spined stickleback (Pungitius pungitius) populations. PLoS One 2011, 6:e28859.
38. Waser W, Sahoo TP, Herczeg G, Merilä J, Nikinmaa M: Physiological differentiation among nine-spined stickleback populations: effects of copper exposure. Aquat Toxicol 2010, 98:188-195.

39. Feulner PG, Chain FJ, Panchal M, Eizaguirre C, Kalbe M, Lenz TL, Mundry M, Samonte IE, Stoll M, Milinski M, et al: Genome-wide patterns of standing genetic variation in a marine population of three-spined sticklebacks. Mol Ecol 2013, 22:635-649.

40. Hohenlohe PA, Bassham S, Etter PD, Stiffler N, Johnson EA, Cresko WA: Population genomics of parallel adaptation in threespine stickleback using sequenced RAD tags. PloS Genet 2010, 6:e1000862.

41. Shikano T, Ramadevi J, Merilä J: Identification of local- and habitat-dependent selection: scanning functionally important genes in nine-spined sticklebacks (Pungitius pungitius). Mol Biol Evol 2010, 27:2775-2789.

42. Bruneaux M, Johnston SE, Herczeg G, Merilä J, Primmer CR, Vasemagi A: Molecular evolutionary and population genomic analysis of the ninespined stickleback using a modified restriction-site-associated DNA tag approach. Mol Ecol 2013, 22:565-582

43. Shikano T, Ramadevi J, Shimada Y, Merilä J: Utility of sequenced genomes for microsatellite marker development in non-model organisms: a case study of functionally important genes in nine-spined sticklebacks (Pungitius pungitius). BMC Genomics 2010, 11:334.

44. Andres JA, Larson EL, Bogdanowicz SM, Harrison RG: Patterns of transcriptome divergence in the male accessory gland of two closely related species of field crickets. Genetics 2013, 193:501-513.

45. Barreto FS, Moy GW, Burton RS: Interpopulation patterns of divergence and selection across the transcriptome of the copepod Tigriopus californicus. Mol Ecol 2011, 20:560-572.

46. Elmer KR, Fan S, Gunter HM, Jones JC, Boekhoff S, Kuraku S, Meyer A: Rapid evolution and selection inferred from the transcriptomes of sympatric crater lake cichlid fishes. Mol Ecol 2010, 19(Suppl 1):197-211.

47. Meyer E, Aglyamova GV, Wang S, Buchanan-Carter J, Abrego D, Colbourne JK, Willis BL, Matz MV: Sequencing and de novo analysis of a coral larval transcriptome using 454 GSFIx. BMC Genomics 2009, 10:219.

48. Parchman TL, Geist KS, Grahnen JA, Benkman CW, Buerkle CA: Transcriptome sequencing in an ecologically important tree species: assembly, annotation, and marker discovery. BMC Genomics 2010, 11:180.

49. Vera JC, Wheat CW, Fescemyer HW, Frilander MJ, Crawford DL, Hanski I, Marden JH: Rapid transcriptome characterization for a nonmodel organism using 454 pyrosequencing. Mol Ecol 2008, 17:1636-1647.

50. Axelsson E, Ratnakumar A, Arendt ML, Maqbool K, Webster MT, Perloski M, Liberg O, Arnemo JM, Hedhammar A, Lindblad-Toh K: The genomic signature of dog domestication reveals adaptation to a starch-rich diet. Nature 2013, 495:360-364.

51. Zhao S, Zheng P, Dong S, Zhan X, Wu Q, Guo X, Hu Y, He W, Zhang S, Fan $W$, et al: Whole-genome sequencing of giant pandas provides insights into demographic history and local adaptation. Nat Genet 2013, 45:67-71.

52. Guo B, Zou M, Gan X, He S: Genome size evolution in pufferfish: an insight from BAC clone-based Diodon holocanthus genome sequencing. BMC Genomics 2010, 11:396.

53. Guo B, Gan X, He S: Hox genes of the Japanese eel Anguilla japonica and Hox cluster evolution in teleosts. J Exp Zool Part B 2010, 314:135-147.

54. Zou M, Guo B, He S: The roles and evolutionary patterns of intronless genes in deuterostomes. Comp Funct Genomics 2011, 2011:680673.

55. Guo B, Zou M, Wagner A: Pervasive indels and their evolutionary dynamics after the fish-specific genome duplication. Mol Biol Evol 2012, 29:3005-3022

56. Guo B, Tong C, He S: Sox genes evolution in closely related young tetraploid cyprinid fishes and their diploid relative. Gene 2009, 439:102-112.

57. Kulathinal RJ, Stevison LS, Noor MA: The genomics of speciation in Drosophila: diversity, divergence, and introgression estimated using low-coverage genome sequencing. Plos Genet 2009, 5:e1000550.

58. Noor MA, Feder JL: Speciation genetics: evolving approaches. Nat Rev Genet 2006, 7:851-861.

59. Yang Z, Bielawski JP: Statistical methods for detecting molecular adaptation. Trends Ecol Evol 2000, 15:496-503.

60. Hurst LD: The $\mathrm{Ka} / \mathrm{Ks}$ ratio: diagnosing the form of sequence evolution. Trends Genet 2002, 18:486.

61. Kumar S, Subramanian S: Mutation rates in mammalian genomes. Proc Natl Acad Sci USA 2002, 99:803-808.

62. Ravi $\mathrm{V}$, Venkatesh B: Rapidly evolving fish genomes and teleost diversity. Curr Opin Genet Dev 2008, 18:544-550. 
63. Drummond DA, Bloom JD, Adami C, Wilke CO, Arnold FH: Why highly expressed proteins evolve slowly. Proc Natl Acad Sci USA 2005, 102:14338-14343.

64. Ding Y, Zhou Q, Wang W: Origins of new genes and evolution of their novel functions. Annu Rev Ecol Evol S 2012, 43:345-363.

65. Hahn MW, Demuth JP, Han SG: Accelerated rate of gene gain and loss in primates. Genetics 2007, 177:1941-1949.

66. Guo B, Wagner A, He S: Duplicated gene evolution following wholegenome duplication in teleost Fish. In Gene Duplication. Edited by Friedberg F. Rijeka, Croatia: InTech; 2011:27-36.

67. Blomme T, Vandepoele K, De Bodt S, Simillion C, Maere S, Van de Peer Y: The gain and loss of genes during 600 million years of vertebrate evolution. Genome Biol 2006, 7:R43.

68. Jones FC, Chan YF, Schmutz J, Grimwood J, Brady SD, Southwick AM, Absher DM, Myers RM, Reimchen TE, Deagle BE, et al: A genome-wide SNP genotyping array reveals patterns of global and repeated species-pair divergence in sticklebacks. Curr Biol 2012, 22:83-90.

69. Margulies M, Egholm M, Altman WE, Attiya S, Bader JS, Bemben LA, Berka J, Braverman MS, Chen YJ, Chen ZT, et al: Genome sequencing in microfabricated high-density picolitre reactors. Nature 2005, 437:376-380.

70. Altschul SF, Madden TL, Schaffer AA, Zhang JH, Zhang Z, Miller W, Lipman DJ: Gapped BLAST and PSI-BLAST: a new generation of protein database search programs. Nucleic Acids Res 1997, 25:3389-3402

71. Hubbard T, Andrews D, Caccamo M, Cameron G, Chen Y, Clamp M, Clarke L, Coates G, Cox T, Cunningham F, et al: Ensembl 2005. Nucleic Acids Res 2005, 33:D447-D453.

72. Kent WJ: BLAT-the BLAST-like alignment tool. Genome Res 2002, 12:656-664

73. Gotz S, Garcia-Gomez JM, Terol J, Williams TD, Nagaraj SH, Nueda MJ, Robles M, Talon M, Dopazo J, Conesa A: High-throughput functional annotation and data mining with the Blast2GO suite. Nucleic Acids Res 2008, 36:3420-3435.

74. Ashburner M, Ball CA, Blake JA, Botstein D, Butler H, Cherry JM, Davis AP, Dolinski K, Dwight SS, Eppig JT, et al: Gene Ontology: tool for the unification of biology. Nat Genet 2000, 25:25-29.

75. Bluthgen N, Brand K, Cajavec B, Swat M, Herzel H, Beule D: Biological profiling of gene groups utilizing Gene Ontology. Genome Inform 2005, 16:106-115.

76. Birney E, Clamp M, Durbin R: GeneWise and genomewise. Genome Res 2004, 14:988-995.

77. Edgar RC: MUSCLE: multiple sequence alignment with high accuracy and high throughput. Nucleic Acids Res 2004, 32:1792-1797.

78. Yang $\mathrm{ZH}$, Nielsen R: Estimating synonymous and nonsynonymous substitution rates under realistic evolutionary models. Mol Biol Evol 2000, 17:32-43.

79. Yang ZH: PAML 4: Phylogenetic analysis by maximum likelihood. Mol Biol Evol 2007, 24:1586-1591.

80. Kimura M: A simple method for estimating evolutionary rates of base substitutions through comparative studies of nucleotide-sequences. J Mol Evol 1980, 16:111-120.

81. Rice P, Longden I, Bleasby A: EMBOSS: the european molecular biology open software suite. Trends Genet 2000, 16:276-277.

82. Yang $\mathrm{ZH}$, Nielsen R: Codon-substitution models for detecting molecular adaptation at individual sites along specific lineages. Mol Biol Evol 2002, 19:908-917.

83. Zhang JZ, Nielsen R, Yang ZH: Evaluation of an improved branch-site likelihood method for detecting positive selection at the molecular level. Mol Biol Evol 2005, 22:2472-2479.

84. Storey JD, Tibshirani R: Statistical significance for genomewide studies. P Natl Acad Sci USA 2003, 100:9440-9445.

85. Li H, Durbin R: Fast and accurate long-read alignment with burrowswheeler transform. Bioinformatics 2010, 26:589-595.

86. Li H, Handsaker B, Wysoker A, Fennell T, Ruan J, Homer N, Marth G, Abecasis G, Durbin R, Proc GPD: The sequence alignment/Map format and SAMtools. Bioinformatics 2009, 25:2078-2079.

87. Lynch M, Sung W, Morris K, Coffey N, Landry CR, Dopman EB, Dickinson WJ, Okamoto K, Kulkarni S, Hartl DL, et al: A genome-wide view of the spectrum of spontaneous mutations in yeast. Proc Natl Acad Sci USA 2008, 105:9272-9277.

88. Mcdonald $\mathrm{JH}$, Kreitman M: Adaptive protein evolution at the Adh locus in Drosophila. Nature 1991, 351:652-654

89. Thornton K: libsequence: a C++ class library for evolutionary genetic analysis. Bioinformatics 2003, 19:2325-2327.
90. Stoletzki N, Eyre-Walker A: Estimation of the neutrality index. Mol Biol Evol 2011, 28:63-70.

91. Rand DM, Kann LM: Excess amino acid polymorphism in mitochondrial DNA: contrasts among genes from drosophila, mice, and humans. Mol Biol Evol 1996, 13:735-748.

92. Thiel T, Michalek W, Varshney RK, Graner A: Exploiting EST databases for the development and characterization of gene-derived SSR-markers in barley (Hordeum vulgare L.). Theor App/ Genet 2003, 106:411-422.

93. Ye J, Fang L, Zheng H, Zhang Y, Chen J, Zhang Z, Wang J, Li S, Li R, Bolund L, et al: WEGO: a web tool for plotting GO annotations. Nucleic Acids Res 2006, 34:W293-297.

doi:10.1186/1471-2164-14-756

Cite this article as: Guo et al:: Genomic divergence between nine- and three-spined sticklebacks. BMC Genomics 2013 14:756.

\section{Submit your next manuscript to BioMed Central and take full advantage of:}

- Convenient online submission

- Thorough peer review

- No space constraints or color figure charges

- Immediate publication on acceptance

- Inclusion in PubMed, CAS, Scopus and Google Scholar

- Research which is freely available for redistribution

Submit your manuscript at www.biomedcentral.com/submit
C) BioMed Central 